\section{The multidisciplinary art and science of cancer care: integrating psycho-oncology}
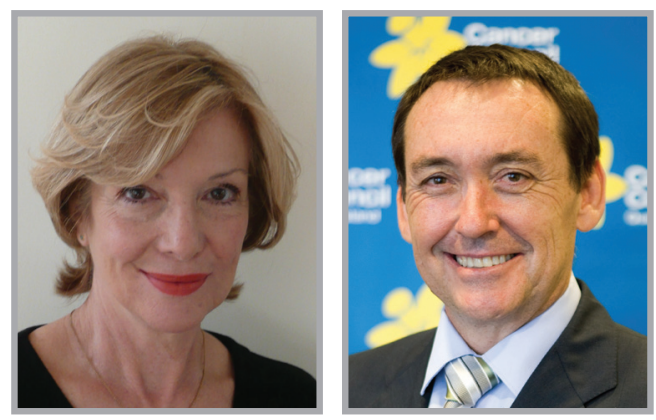

\author{
"There is still too little being \\ done to integrate \\ psycho-oncology into the \\ spectrum of cancer control \\ and care."
} \section{Future ONCOLOGY}

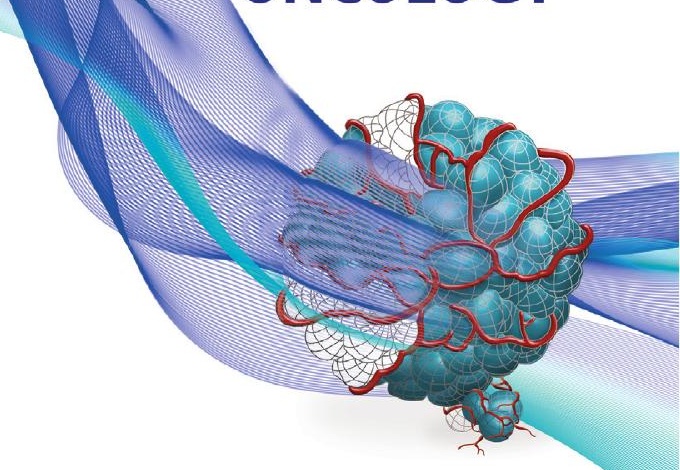

Maggie Watson ${ }^{*, 1} \&$ Jeffrey Dunn $n^{2,3,4,5}$
First draft submitted: 8 August 2016; Accepted for publication: 2 September 2016; Published online: 14 September 2016

\section{What is psycho-oncology?}

Psycho-oncology is an evolving subspecialty generally focusing on psychological, social, behavioral and psychiatric aspects of oncology. The science of psycho-oncology, which has developed particularly well over the past 30 years, aims to provide an evidence base that could give direction to improvement of multidisciplinary cancer care by encompassing and integrating mental healthcare and the psychological sciences.

Yet, psycho-oncology also includes a broader spectrum of interests and scientific activity; it encompasses an understanding of behavioral, social and lifestyle factors that contribute to increased risk of developing cancer. Furthermore, it provides insights into survivorship issues affecting people experiencing the late effects of cancer treatments and, in some cases, continuing physical and psychological disability. Until now, psycho-oncology has often struggled to become integrated with these aspects of cancer: a soft science that remained of low priority. What follows now are some specifics on how it might be possible to better integrate psycho-oncology into cancer care.

\section{Psycho-oncology in routine multidisciplinary cancer care}

Areas that are providing improvements to patient quality of life include the introduction of routine assessment and screening for distress and coping. The International Psycho-Oncology Society [1] has advocated that screening for distress should be considered the sixth vital sign and incorporated into regular comprehensive cancer care [2]. Different methods of distress screening have been developed and evaluated to assist busy oncology staff in assessing patients' psychosocial needs. The main impetus has been toward having professionals, directly involved in cancer care; make use of these

\section{KEYWORDS}

- behavioral • cancer

- cost-effectiveness oncology

- distress screening • prevention

- psychological intervention

- psycho-oncology • psychosocial

- survivorship

'Pastoral \& Psychological Care, Compton House, The Royal Marsden NHS Trust, Downs Road, Sutton, Surrey, 
“The increasing focus on individual responsibilities

to reduce cancer risk and the absent voice of

psycho-oncology in cancer

prevention, serves as an

impetus for involvement of

our discipline in creating

and revising national

cancer control plans.” brief methods of distress screening on a regular basis.

In the UK, the NICE [3] has operationalized psychological care within a four-tier stepped care model. Patients with psychosocial care needs can be evaluated and treated using this approach moving from low through to high needs and level of care skills offered depend on further clinical assessment and triage.

In brief, stepped care models are where screening for distress or need is applied, and services targeted and delivered according to need. In this way care can be stepped up if distress or need fails to resolve. A related but different concept is that of low intensity care; an approach that has improved access as its central value [4]. Specifically, low intensity care aims to deliver evidence-based care in an equitable fashion and so utilizes novel delivery approaches (such as remote or nonspecialist delivery) to aid widespread dissemination. Several groups have operationalized these approaches in a range of settings. In one of the earliest models, and the first from a community setting, Hutchison and colleagues [5] proposed a tiered model that applied screening to identify patient need. Once identified, they triaged patients or carers to a level of care where the intensity of the intervention is matched to the depth of distress and the complexity of presenting problem

\section{Psychological intervention in cancer care: what works?}

Delivery of psycho-oncology care at the lowest level of need can be provided by cancer clinical staff with limited psychological skills training. A few studies have shown that nonpsycho-oncologists can be trained to use specific formal psychological therapies, for example, cognitive-behavioral therapy (CBT) [6,7]. The minority of patients with more complex psychosocial needs can be referred to psycho-oncologists, with advanced mental health skills, who will implement proven high-level therapies. Integrating these high level mental health specialists, into locations where cancer is treated, is essential to providing good seamless comprehensive care. Cancer patients should not have to attend external mental health units to receive this care, unless they have serious problems such as suicide risk requiring mental health management facilities. The amount of evidence on efficacy of psychological intervention for adult patients is substantial. There are a number of metaanalyses and supra-meta-analyses [8-13]. Newell and colleagues [10] provide one of the better reviews and conclude that group therapy, education, counseling and CBT offer 'most promise' for medium- and long-term benefits. More recently the UK NICE $[14,15]$ review of depression in adults with chronic health problems, recommended the use of group CBT, individual CBT and computer-delivered CBT, as the treatments of choice for depression in patients with chronic health problems, given the weight of evidence on efficacy.

\section{How much does this all cost?}

Increasingly healthcare providers are being asked to offer evidence of cost effectiveness to justify the introduction or continuation of healthcare services. To date, there has been a lack of data on cost-effectiveness in the field of psycho-oncology, although this is rapidly changing. Simpson and colleagues [16] found that in a randomized controlled study, a brief group psycho-educational intervention for breast cancer patients, when compared with a wait-list control group saved healthcare system costs. The evidence on cost effectiveness is slowly accumulating [17-20]. Recently Dieng and colleagues [21] undertook a systematic review of psychosocial interventions in cancer, concluding that cognitive-behavioral interventions in particular, represent good value for money in cancer care. However, several limitations in the literature were noted, including the need for more clarity in how economic questions are posed, a more comprehensive approach to measuring cost, and the need for further application of cost utility measures to address the issue of estimating quality-adjusted life years. Economic analyses have been usefully applied to examine how care models or approaches can be used efficiently. As an example, Duarte et al. [22] examined the cost-effectiveness of collaborative treatment for cancer patients with depression and reported this approach was likely cost effective at the current thresholds used by NICE. This collaborative treatment model applied a multicomponent, manualized treatment program that integrates specialist depression treatment within oncology and primary care. The principal care deliverers in this approach are nurses, who work under the supervision of psychiatrists, with patients referred for psychiatric consultation if they fail to respond to treatment. In a community-based setting a trial with high distress cancer patients and carers found a single nurse counselor session 
to be as effective as five psychologist delivered sessions of CBT [23]. However, an economic analysis by Chatterton et al. [24] found that for those patients and carers at the higher distress levels, the psychologist delivered intervention was likely more cost effective in terms of later health sector cost savings. What is clear from these studies is that the overall care framework is important in determining how efficiently overall resources are utilized.

\section{Psycho-oncology \& cancer prevention}

A recent commentary [25] indicated that approximately $30 \%$ of cancer deaths are attributable to modifiable lifestyle, behavioral and psychosocial risk factors. With effective intervention these risk behaviors can be reduced and potentially half of all cancers prevented [26]. A recent editorial highlights the role of social and cultural inequalities in cancer outcomes and calls for more psychosocial research to be a future priority [27]. Psycho-oncology is not routinely included in reference to primary and secondary cancer prevention and available behavioral interventions are not being fully utilized. The increasing focus on individual responsibilities to reduce cancer risk and the absent voice of psycho-oncology in cancer prevention, serves as an impetus for involvement of our discipline in creating and revising national cancer control plans.

\section{Here is an agenda for change}

There is still too little being done to integrate psycho-oncology into the spectrum of cancer control and care. A shift is needed, along with a willingness to see beyond the immediate pressures to deal with cancer as a biological disease. We suggest, the following will provide a good structure for positive and productive change toward a multidisciplinary comprehensive approach to cancer:

- Bring psychosocial, behavioral and psychooncology scientists more firmly and directly into the national and global cancer prevention programs. There are still too few involved;
- Make psychosocial care of people with cancer a routinely accepted part of multidisciplinary cancer care: assess distress and support needs as the sixth vital sign;

- Change approaches to training in oncology. Psycho-oncologists have both highly specialist mental health skills to offer and a wonderful history of giving away their skills to other professionals. By offering training to improve psychosocial care skills across the board, we can allow more patients to receive good psychosocial care;

- Ensure psycho-oncologists are integrated into cancer rehabilitation programs;

- Recognize that psycho-oncologists contribute in important ways to evidence based cancer care. Invite them into research committees; give them a role to play in the development of national and international policies on cancer care; increase their contribution to oncology training programs and multidisciplinary research;

- Support and encourage the development of psycho-oncology as a clinical science; by doing so you support an improvement in evidence-based care, and in the quality of life of those patients badly affected by cancer both physically and emotionally.

\section{Financial \& competing interests disclosure}

The authors have no relevant affiliations or financial involvement with any organization or entity with a financial interest in or financial conflict with the subject matter or materials discussed in the manuscript. This includes employment, consultancies, honoraria, stock ownership or options, expert testimony, grants or patents received or pending, or royalties.

No writing assistance was utilized in the production of this manuscript.

\section{Open access}

This work is licensed under the Creative Commons Attribution-NonCommercial 4.0 Unported License. To view a copy of this license, visit http://creativecommons.org/ licenses/by-nc-nd/4.0/

\section{References}

1 International Psycho-Oncology Society. www.ipos-society.org

2 Holland J, Watson M, Dunn J. The IPOS new international standard of quality cancer care: integrating the psychosocial domain into routine care. Psychooncology 20, 677-680 (2011).

3 NICE. Guidance on cancer services improving supportive and palliative care for adults with cancer (2004).

www.nice.org.uk
4 Bennett-Levy J, Richards DA, Farrand P et al. Low Intensity CBT Interventions: $A$ Revolution in Mental Health Care. Oxford Guide to Low Intensity CBT Interventions. Oxford University Press, Oxford, UK, 3-18 (2010) 
5 Hutchison S, Steginga SK, Dunn J. A tiered model of psychosocial intervention in cancer: translating psychosocial care practice guidelines into action. Psychooncology 15(6), 541-546 (2006).

6 Moorey S, Cort E, Kapari M et al. A cluster randomized controlled trial of cognitive behaviour therapy for common mental disorders in patients with advanced cancer. Psychol. Med. 39, 713-723 (2009).

7 Pitceathly C, Maguire P, Fletcher I et al. Can a brief psychological intervention prevent anxiety or depressive disorders in cancer patients? A randomised controlled trial. Ann. Oncol. 20(5), 928-934 (2009).

8 Fors EG, Bertheussen GF, Thune I et al. Psychosocial interventions as part of breast cancer rehabilitation programs? Results from a systematic review. Psychooncology 20, 909-918 (2011).

9 Naaman SC, Radwan K, Fergusson D, Johnson S. Status of psychological trials in breast cancer patients: a report of three meta-analyses. Psychiatry 72, 50-69 (2009).

10 Newell SA, Sanson-Fisher RW, Savolainen NJ. Systematic review of psychological therapies for cancer patients: overview and recommendations for future research. J. Natl Cancer Inst. 94 (8), 558-584 (2002).

11 Rehse B, Pukrop R. Effects of psychosocial interventions on quality of life in adult cancer patients: meta-analysis of 37 published controlled outcome studies. Patient Educ. Counsel. 50, 179-186 (2003).

12 Osborn R, Demoncada A, Feuerstein M. Psychosocial interventions for depression, anxiety, and quality of life in cancer survivors: meta-analyses. Int. J. Psychiatry Med. 36, 13-34 (2006).

13 Matthews H, Grunfeld E, Turner A. The efficacy of interventions to improve psychosocial outcomes following surgical treatment for breast cancer: a systematic review. Psychooncology (2016) (In Press).

14 Depression in Adults with a Chronic Physical Health Problem: Treatment and Management. National Clinical Practice Guideline Number 91. National Collaborating Centre for Mental Health commissioned by the NICE (2009). www.nice.org.uk/Guidance/cg91

15 NICE. Technology Appraisal No. 51. Computerised Cognitive Behaviour Therapy (2006). www.nice.org.uk/guidance/ta97

16 Simpson JSA, Carlson LE, Trew ME. Effect of group therapy for breast cancer on healthcare utilization. Cancer Pract. 9, 19-26 (2001).

17 Gordon LG, Scuffham P, Battistutta D, Graves N, Tweeddale M, Newman B. A cost-effectiveness analysis of two rehabilitation support services for women with breast cancer. Breast Cancer Res. Treat. 94, 123-133 (2005).

18 Lemieux J, Topp A, Chappell H, Ennis M, Goodwin PJ. Economic analysis of psychosocial group therapy in women with metastatic breast cancer. Breast Cancer Res. Treat. 100, 183-190 (2006).

19 Mandelblatt JS, Cullen J, Lawrence WF et al. Economic evaluation alongside a clinical trial of psycho-educational interventions to improve adjustment to survivorship among patients with breast cancer. J. Clin. Oncol. 26, 1684-1690 (2008).
20 Sabariego C, Brach M, Herschbach P, Berg P, Stucki G. Cost-effectiveness of cognitive-behavioral group therapy for dysfunctional fear of progression in cancer patients. Eur. J. Health Econ. 12, 489-497 (2011).

21 Dieng M, Cust AE, Kasparian NA, Mann GJ, Morton RL. Economic evaluations of psychosocial interventions in cancer: a systematic review. Psychooncology doi:10.1002/ pon.4075 (2016) (Epub ahead of print).

22 Duarte A, Walker J, Walker S et al. Cost-effectiveness of integrated collaborative care for comorbid major depression in patients with cancer. Psychosom. Res. 79, 465-470 (2015).

23 Chambers SK, Girgis A, Occhipinti S et al. A randomised trial comparing two low intensity psychological interventions for distressed cancer patients and caregivers. Oncol. Nurs. Forum 41, 256-266 (2014).

24 Chatterton ML, Chambers S, Occhipinti S et al. Economic evaluation of a psychological intervention for high distress cancer patients and carers: costs and quality-adjusted life years. Psychooncology 25, 857-864 (2016).

25 Dunn J, Holland J, Hyde MK, Watson M. Psycho-oncology and primary prevention in cancer control plans: an absent voice? Psychooncology 24, 1338-1345 (2015).

26 Vineis P, Wild CP. Global cancer patterns: causes and prevention. Lancet 383, 549-557 (2014).

27 Ashley L, Lawrie I. Tackling inequalities in cancer care and outcomes: psychosocial mechanisms and targets for change. Psychooncology (2016) (In Press). 